$\succ$ sell emerging technology and intellectual property at a reasonable cost to support product development. Academics must realize that just sending off a progress report after cogitating in the laboratory is not useful for a company.

On the other side, industry must appreciate that universities are not buffets of fully mature technologies there for the taking, free of charge. Companies should expect to invest time and money to move from an academic prototype to a commercial product. Industrialists must acknowledge that repeated measurements of reproducibility, lifetime and reliability are difficult to fit into the academic framework of constant innovation, and are not the best use of researchers' skills.

What both partners need from each other must be made clear at the outset. As Olav Solgaard, an electrical engineer at Stanford, explained: successful collaborations require leaders on both sides to manage expectations and to set sensible ground rules. Both must agree on outputs such as publications, especially when $\mathrm{PhD}$ students are involved. Simple and direct approaches are necessary. Many universities in China, for example, are involved in managing the companies with which they collaborate ${ }^{8}$. Others find that integrating industrial researchers into university laboratories is effective.

Hiroshi Toshiyoshi at the University of Tokyo, who has a long record of developing microelectromechanical systems and collaborating with industry told me how he likes to operate: "I like to ask my partner company to send their researchers to my group, where I give theoretical and on-the-job training for a year or two. We may not be able to deliver immediate results, but the company will obtain longlasting competence."

So, set aside some lab space, fill it with recent graduates and company researchers, shake well and let the nutty academic idea evolve into the useful industrial prototype.

Hans Zappe is chair of micro-optics in the Department of Microsystems Engineering, University of Freiburg, Germany. e-mail:zappe@imtek.uni-freiburg.de

1. Okamuro, H. \& Nishimura, J. J. Technol. Transf. 38, 273-301 (2013).

2. Grimpe, C. \& Fier, H. J. Technol. Transf. 35, 637-650 (2010).

3. Czarnitzki, D., Glänzel, W. \& Hussinger, K. Res. Eval. 16, 311-319 (2007).

4. Bradley, S. R., Hayter, C. S. \& Link, A. N. Technol. Transf. 38, 349-381 (2013)

5. Kenney, M. \& Goe, W. R. Res. Policy 33, 691-707 (2004)

6. Perkmann, M. et al. Res. Policy 42, 423-442 (2013).

7. Marazita, C. F. Technol. Forecast. Soc. Change 39, 397-410 (1991)

8. Wu, W. World Dev. 35, 1075-1093 (2007).

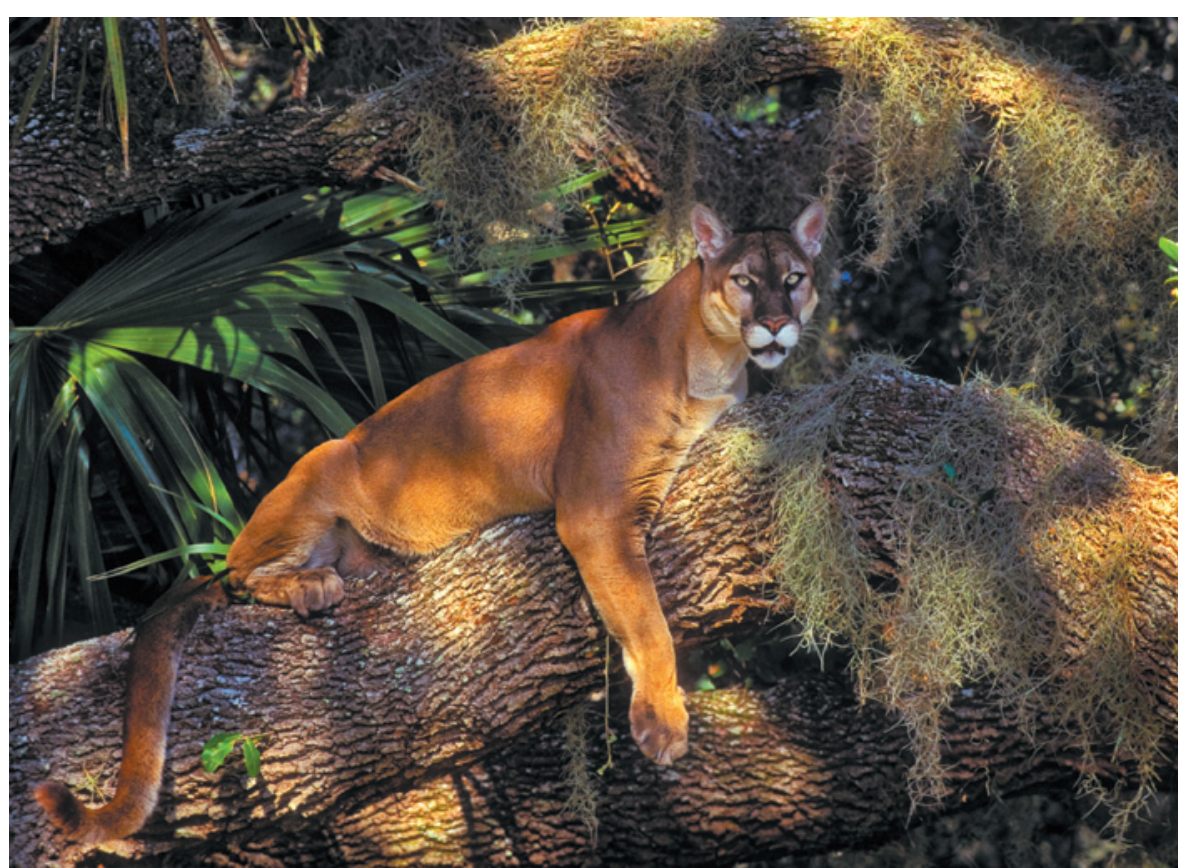

An endangered Florida panther population was bolstered through hybridization with a related subspecies - a technique that could be refined using genomic tools.

\title{
Gene tweaking for conservation
}

\section{It is time to weigh up the pros and cons of using genetic engineering to rescue species from extinction, say Michael A. Thomas and colleagues.}

$\mathrm{E}$ ven the most conservative estimates predict $^{1}$ that $15-40 \%$ of living species will be effectively extinct by 2050 as a result of climate change, habitat loss and other consequences of human activities. In the face of such drastic losses, scientists are debating the pros and cons of various, and often controversial, interventions. These include moving populations to help track hospitable habitats, and reinstating keystone species those that have a large effect on ecosystem structure and function, such as top-level predators - into areas where they have long been absent ${ }^{2,3}$. Even the revival of species that have recently gone extinct is being explored.

So far, an increasingly viable (and potentially less risky) option, which we call facilitated adaptation, has been little discussed. It would involve rescuing a target population or species by endowing it with adaptive alleles, or gene variants, using genetic engineering.

Over the past 30 years, genetic engineering in agriculture has received substantial attention. Today, $12 \%$ of arable land worldwide is planted with genetically modified (GM) crops; the GM seed market alone is valued at US\$15 billion. As techniques become ever more sophisticated, more possibilities will open up.

We believe that these combined factors mean that conservationists will almost certainly be tempted to apply genetic engineering to safeguard biodiversity. Facilitated adaptation might be less logistically challenging than moving entire populations, and less fraught with ecological and socioeconomic complications - relocation could introduce harmful invasive species, for example, or unleash outbreaks of disease. But facilitated adaptation is likely to be beset with other challenges and pitfalls. Now is the time to consider what those might be.

\section{THREE OPTIONS}

There are at least three ways to avert extinction using facilitated adaptation. First, animals or plants from a threatened 
$\checkmark$ population could be crossed or hybridized with individuals of the same species from better-adapted populations to introduce beneficial alleles into the threatened population. Second, specific alleles drawn from a well-adapted population could be directly transferred into the genomes of threatened populations of the same species. And third, genes taken from a well-adapted species could be incorporated into the genomes of endangered individuals of a different species. Each approach carries its own set of challenges, complications and risks.

\section{BACK FROM THE BRINK}

Conservationists have already tried hybridizing individuals to aid the recovery of vulnerable populations. For instance, in an isolated population of the viper Vipera berus in Sweden, the number of inviable offspring produced as a result of inbreeding plummeted when male vipers from a healthy population were introduced ${ }^{4}$. Similarly, the size of a remnant Florida panther (Puma concolor coryi) population effectively increased by $100 \%$ after conservationists introduced eight cats of a related subspecies, Puma concolor stanleyana, from Texas. Heterozygosity, a measure of genetic variation, also rose in the Florida population, from around $18 \%$ to more than $25 \%$ in 12 years ${ }^{5}$.

This practice of injecting lost genetic diversity into a threatened population could be refined using genomic tools. For instance, conservationists could screen potential donor populations for individuals with alleles that would, say, yield physiological tolerance of warmer temperatures, or resistance to a catastrophic disease.

The potential risks of this approach, as with relocating entire populations, include the introduction of wildlife diseases, the dilution of locally adaptive alleles and the disruption of co-adapted gene complexes that impart advantages crucial in the threatened population's local habitat.

Success with the second approach directly transferring specific alleles drawn from a well-adapted population into individuals from a threatened population would similarly depend on introducing enough 'better-adapted' individuals with a sufficient selective advantage to increase the mean fitness of the threatened population. This would require conservationists first to work out which genes are controlling relevant adaptive traits. Earlier this year, aquaculture researchers identified ${ }^{6}$ alleles associated with heat tolerance in a commercial rainbow trout, Oncorhynchus mykiss. These gene variants could potentially be inserted into the genomes of fish eggs or embryos in populations threatened by rising water temperatures because of climate change.

This strategy has promise if one or a few genes have a drastic impact on the trait of interest. For instance, some amphibians are resistant to the fungal disease chytridiomycosis, which has been linked to massive population declines and several extinctions. If one or a few genes are found to increase resistance, they might make excellent targets for transfer.

This form of genetic engineering is probably the least risky of the approaches described here, because it involves the

"Well-
adapted
combinations
of alleles
could be
disrupted."
movement of alleles within the same species, without the attendant risks of moving diseases or parasites. But again, the challenge is that numerous genes tend to be associated with complex traits. Interactions between genes and the environment, and between introduced alleles and existing genetic diversity, will also be difficult to predict. And, as with the hybridization approach, well-adapted combinations of alleles could be disrupted.

The third transgenic approach - transferring genes between different species - has been used for more than a decade to improve resistance to crop stressors, including drought and extreme temperatures $^{7}$. Certain genes from rice and the cress plant Arabidopsis, for instance, make tomato plants more tolerant of cold temperatures. A few years ago, developmental biologists produced mice with exceptionally long digits by replacing a limb-specific transcriptional enhancer of the mouse $\operatorname{Pr} x 1$ gene locus with the homologous bat sequence ${ }^{8}$. Conservationists could use similar approaches to explore the use of other genes, to augment, for example, resistance to white-nose syndrome, a rapidly spreading and deadly fungal disease in North American bats.

The effects of moving genetic variants between species, however, are likely to be even harder to predict than those of transferring variants within species, and a major concern is that such an approach could bring unintended and unmanageable consequences.

\section{PRESERVATION PROGRAMMES}

To begin the process of identifying adaptive genes and alleles, and predicting how they will behave in the target genomic and environmental contexts, conservationists can tap several resources. Natural history museum and herbarium collections worldwide, for example, can provide basic data on biogeography and genetics for many animal and plant species.

Large-scale preservation projects could provide the actual tissues needed to explore adaptations among organisms in different ecological contexts. One such project is the
Global Genome Biodiversity Network - an effort to preserve and provide open access to genomic information and DNA samples from various collections, including those of the Smithsonian National Museum of Natural History in Washington DC (see www.mnh.si.edu/ggi).

Ultimately, successful facilitated adaptation will require unprecedented collaboration between organismal, ecological and molecular biologists and climate scientists ${ }^{9}$. Biorepositories - such as seed banks, natural history museums and zoological parks, including the Frozen Zoo at San Diego Zoo in California, which houses around 9,000 frozen cell samples from endangered species - will need to be integrated with advances in biotechnology and efforts to explore the genomic mechanisms underlying adaptive traits associated with climate change ${ }^{10}$, catastrophic diseases and so on.

Facilitated adaptation will also require a change in people's views about biodiversity conservation and its ethics, practices and impact on society. Even moving members of the panther subspecies from Texas into the Florida panther's ecosystem incited misgivings over the appropriateness of meddling directly with organisms rather than with their habitat.

A serious concern is that even the possibility of using genetic-engineering tools to rescue biodiversity will encourage inaction with regard to climate change. Before genetic engineering can be seriously entertained as a tool for preserving biodiversity, conservationists need to agree on the types of scenario for which facilitated adaptation, managed relocation and other adaptation strategies might be appropriate, and where such strategies are likely to fail or introduce more serious problems.

For some species, facilitated adaptation could turn out to be the only viable remedy.

Michael A. Thomas is professor of biology at Idaho State University in Pocatello, Idaho. Gary W. Roemer, C. Josh Donlan, Brett G. Dickson, Marjorie Matocq, Jason Malaney.

e-mail:mthomas@isu.edu

1. Thomas, C. D. \& Williamson, M. Nature $\mathbf{4 8 2}$, E4-E5 (2012).

2. Donlan, J. et al. Nature 436, 913-914 (2005).

3. Hoegh-Guldberg, O. et al. Science 321, 345-346 (2008).

4. Madsen, T., Stille, B. \& Shine, R. Biol. Conserv. 75, 113-118 (1996).

5. Johnson, W. E. et al. Science 329, 1641-1645 (2010).

6. Rebl, A. et al. Mar. Biotechnol. 15, 445-460 (2013).

7. Zhang, J. et al. In Vitro Cell. Dev. Biol. Plant 36 108-114 (2000).

8. Cretekos, C. J. et al. Genes Dev. 22, 141-151 (2008).

9. Redford, K. H., Adams, W. \& Mace, G. M. PLoS Biol. 11, e1001530 (2013).

10.Whitehead, A. J. Exp. Biol. 215, 884-891 (2012). 